\title{
Effects of oblique energetic bombardment on the morphology and microstructure of triode ion plated titanium films
}

\author{
K. R. Gunasekhar and M. Ghanashyam Krishna \\ Instrumentation and Services Unit, Indian Institute of Science, Bangalore-560 012, India \\ A. R. Raju and K. N. Krishna \\ Materials Research Center, Indian Institute of Science, Bangalore-560 012, India \\ S. Mohan \\ Instrumentation and Services Unit, Indian Institute of Science, Bangalore-560 012, India
}

\begin{abstract}
The effect of angle of incidence of ions, varied between $45^{\circ}$ and $90^{\circ}$, generated in a triode ion plating system on the morphology and microstructure of titanium films, has been investigated. It has been found that intensity of reflections, lattice parameters, stress, and grain size are strong functions of the angle of incidence of the ions. The same properties were studied for their variation with bias voltage between 0 and $2 \mathrm{kV}$ at a constant substrate bias current of $10 \mathrm{~mA}$. It was found that the intensity of the (002) reflection increases with an increase in angle of ion incidence up to $60^{\circ}$. The lattice parameter and stress reach a minimum while the grain size goes through a maximum at this value. All the films show a tensile stress independent of deposition parameters. To isolate the effects due to ion bombardment the films have been coated in high vacuum, in a diode mode, and finally the triode configuration. It has been conclusively found that the angle of incidence of ions is very important in controlling properties and growth of the films.
\end{abstract}

\section{INTRODUCTION}

Plasma assisted processing of thin films has gained considerable importance due to the number of property control variables that such a process provides. Thus, properties of films can be tailored by varying ion energies, current densities, ion species, the angle of incidence, etc. either independently or with the variables acting in conjunction. While the effect of the first three parameters on properties of thin film materials has been extensively investigated and reported, the effect of the angle of incidence of energetic particles on the properties of films has only recently been the subject of work. It was earlier assumed that the angle of incidence effects was similar to an increase in pressure during synthesis and was therefore not a subject of much interest. With the advent of ion sources, however, it has been found that the angle of incidence can be varied independent of working pressure and consequently used as an added variable for film property control. ${ }^{1-12}$

The effect of an oblique incidence of ions on the properties of metal films has received considerable attention in recent years. However, most of this work is related to ion assisted deposition, and there is very limited work on these effects in ion plating. In a recent work Gunasekhar et al. ${ }^{13}$ have found that diode ion plated titanium films also show anisotropy in their structural properties. The present investigation has been carried out to establish the nature of growth in obliquely incident ion bombarded titanium films prepared using a triode ion plating configuration. The present work aims to investigate the possibility of using the angle of incidence to control structure and related properties such as stress, grain size, and lattice parameter in ion plated Ti films and their microstructure.

\section{EXPERIMENT}

The films were coated in a differentially pumped chamber described in detail earlier. ${ }^{13,14}$ The starting material Ti was evaporated from a $6 \mathrm{~kW}$ electron beam gun. The distance between the source and substrates was $15 \mathrm{~cm}$, and a special fixture was used to introduce different angles of incidence. The substrates were $0^{\circ}, 10^{\circ}, 20^{\circ}, 30^{\circ}$, and $45^{\circ}$ to the vapor source and $90^{\circ}, 80^{\circ}, 70^{\circ}, 60^{\circ}$, and $45^{\circ}$ to the thermionic filament. The substrate temperature was measured using a iron-constantan thermocouple, before and at the end of the process. The maximum temperature rise at the substrate was found to be $175^{\circ} \mathrm{C}$ at the highest power conditions.

Films were coated onto $\mathrm{Cu}$ substrates at bias voltages of $-1,-1.5$, and $-2 \mathrm{kV}$ at a substrate bias current of $10 \mathrm{~mA}$ and a background Ar pressure of $2 \mathrm{~Pa}$ in the triode mode. They were also coated in the diode mode at a bias voltage of $-2 \mathrm{kV}$, the substrate bias current was $2 \mathrm{~mA}$ and without a process gas in the pressure range $0.001 \mathrm{~Pa}$ without bias. This was done to differentiate between the causes for the observed behavior. The resulting films were characterized for their structure using a (PW 1070 Phillips) powder x-ray diffractometer. The residual thin film stress, grain size, and lattice parameter were calculated using standard expressions described elsewhere. ${ }^{15}$ The scanning electron micrographs were obtained from a Cambridge Stereoscan S-360 scanning electron microscope. Schematic views of the chamber and the substrate fixture are shown in Figs. 1(a) and 1(b), respectively. 


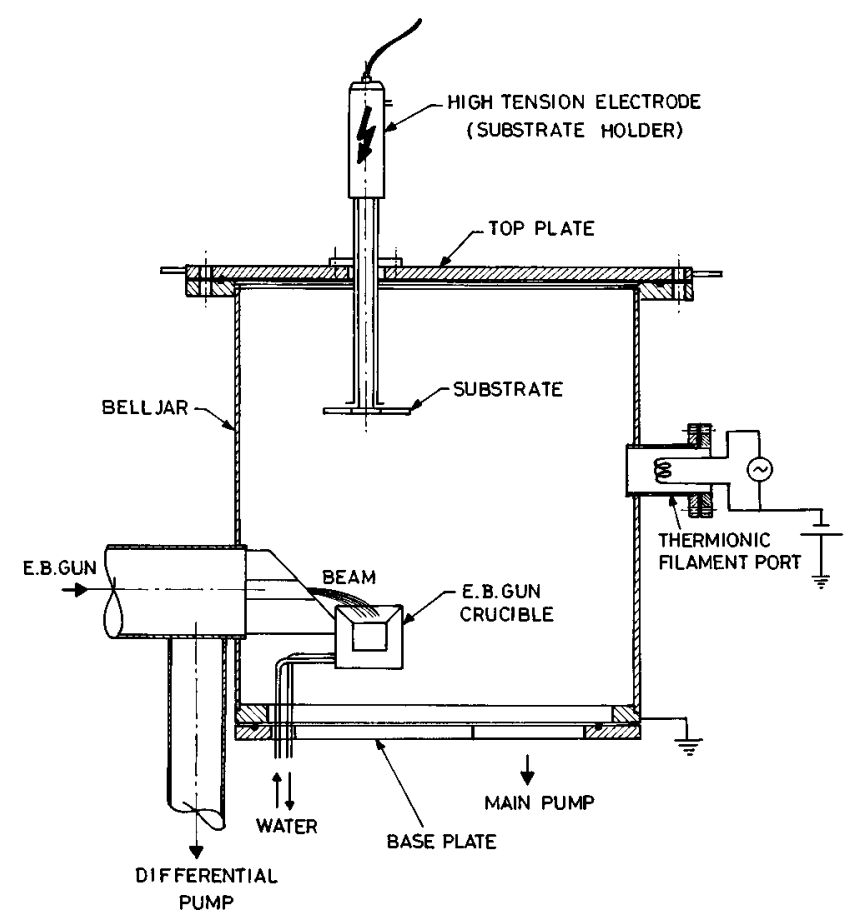

(a)
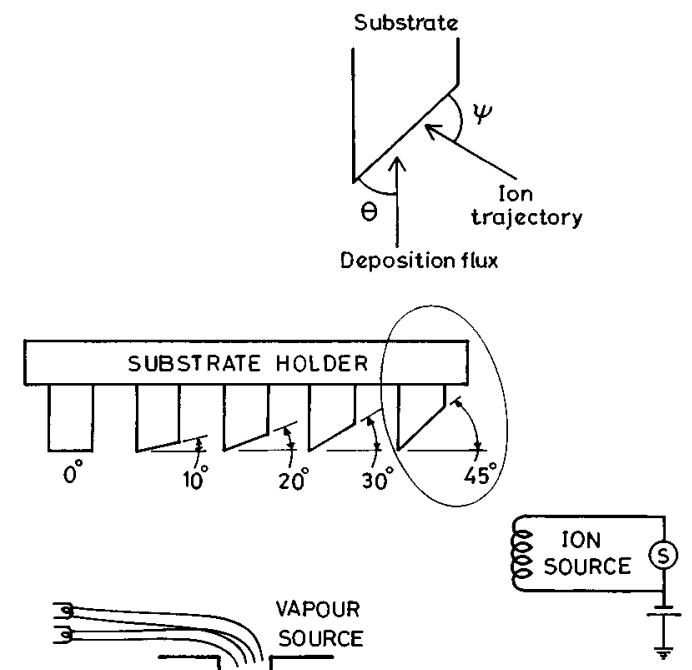

(b)

FIG. 1. Schematic view of (a) the experimental chamber and (b) the substrate holder.

\section{RESULTS AND DISCUSSION}

\section{A. Results}

The $\mathrm{x}$-ray diffraction patterns of the films deposited in the pressure range of $0.001 \mathrm{~Pa}$ without bias, (i.e., electron beam evaporation) at different angles of vapor incidence are shown in Fig. 2(a). The x-ray diffraction patterns reveal that the films show a preferred (002) orientation and that the film deposited normal to the vapor showed a very intense peak. The peak intensity also appeared to be dependent on the angle of ion incidence. It dropped to a very low value at $80^{\circ}$ ion incidence and increased at $70^{\circ}$, reaching a maximum at

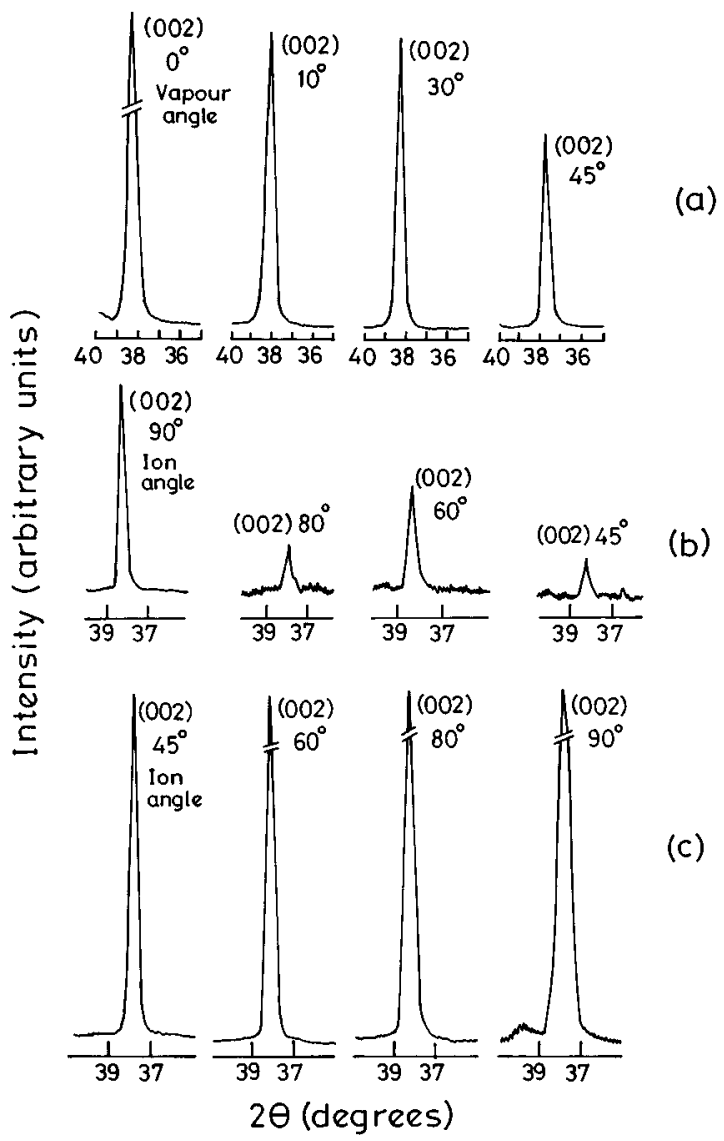

FIG. 2. X-ray diffraction patterns of the films deposited at different angles of (a) vapor incidence without bias, (b) ion incidence at a bias voltage of $1 \mathrm{kV}$, and (c) ion incidence at a bias voltage of $2 \mathrm{kV}$.

$60^{\circ}$, and decreasing beyond that. The diode ion plated films showed the same behavior, with only the absolute intensities being higher. The triode ion plated films, on the other hand, exhibited more drastic changes in intensity of the (002) reflection [Figs. 2(b) and 2(c)].

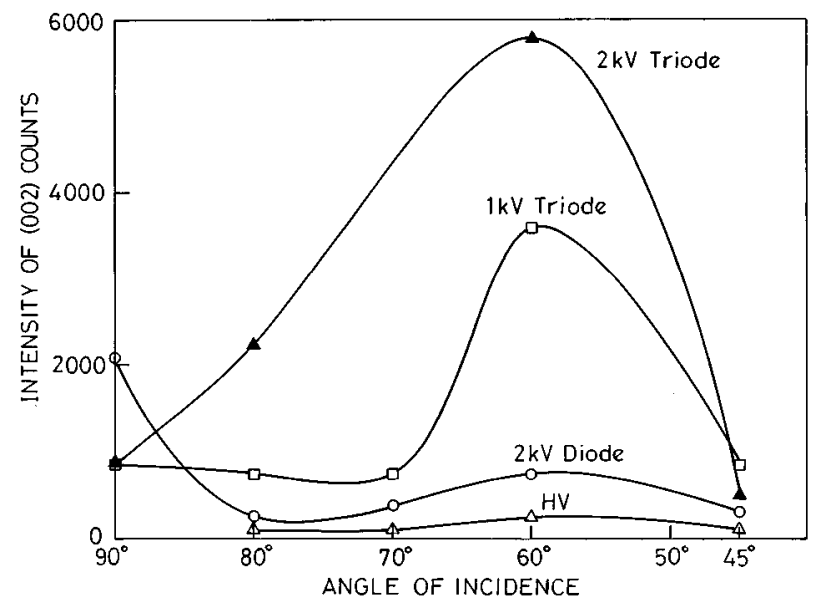

FIG. 3. Variation in intensity of the (001) reflection with the angle of ion incidence at given bias voltages. 
To better understand the change in intensity of the (002) peak with angle of ion incidence, it has been plotted in Fig. 3 as a function of the angle of incidence. It is evident from this figure that as the angle of ion incidence increases from $45^{\circ}$ to $90^{\circ}$, the intensity seems to show a peak around $60^{\circ}$, which corresponds to $30^{\circ}$ vapor incidence. The intensity of the peak increases with an increase in bias voltage as well as substrate current. This behavior is very similar to the sputter yield of titanium with angle of ion incidence observed by Oeschner. ${ }^{16}$ It can thus be stated that the intensity variations are a direct consequence of the variation in the sputter yield of titanium with the angle of ion incidence. The decrease in the intensity at values higher than the peak for all the films can be attributed to the reflection coefficient of the ions dominating over the sputter yield.

It is interesting to note that all the films show a preferred (002) orientation. In metal films deposited using other means of ion bombardment this has been attributed to the difference in sputtering yields of grains which are aligned in the direction of ion bombardment resulting in channeling and hence lower sputtering yield and those which are misaligned. The difference in sputtering yields in the two cases are as high as a factor of 5 for some materials. ${ }^{17}$ In an extensive study done on $\mathrm{Nb}$ it has been found that the degree of orientational order is a strong function of the ion/atom arrival ratio. ${ }^{6-8}$ Fabis, Cooke, and McDonough ${ }^{10}$ have also studied the effect of varying the substrate orientation to ion incidence in a sputtering environment and found that the structure is very strongly dependent on the angle of incidence. The grain sizes observed in the present cases indicate a restricted fiber grain growth in the films, although it must be stated that the values obtained from x-ray diffraction studies are not very accurate. The effect of substrate temperature has been considered insignificant here, since the temperature did not rise to very high values during deposition. Yu et al. ${ }^{7,8}$ have also found that a substrate temperature of $250{ }^{\circ} \mathrm{C}$ does not induce very drastic changes in $\mathrm{Nb}$ films in contrast to those deposited using ion assisted deposition.

The lattice parameter variation with angle of ion incidence is plotted in Fig. 4. The $c$ parameter is seen to approach that of the bulk between $60^{\circ}$ and $70^{\circ}$ ion incidence angle and increase above and below that. This clearly indicates the anisotropic growth that results from such bombardment. That the growth is a consequence of the triode configuration is amply clear from the fact that only in the case of high vacuum evaporation $(0 \mathrm{kV})$ do the films show a bulklike $c$ parameter and as bias is applied, the anisotropy increases. This would then extend to stresses also as they are directly related to the shifts in the interplanar spacings of the films (Fig. 5). It is quite clear that stresses also go through a minimum as in the case of the lattice parameter. Two important observations can be made from these figures: (1) all the films approach the bulk value of the lattice parameter at $\sim 60^{\circ}-70^{\circ}$ ion incidence angle and (2) they show only tensile stress independent of deposition parameters. The dashed curves represent values obtained from the grain boundary relaxation model $^{18}$ to be discussed later. Significantly this

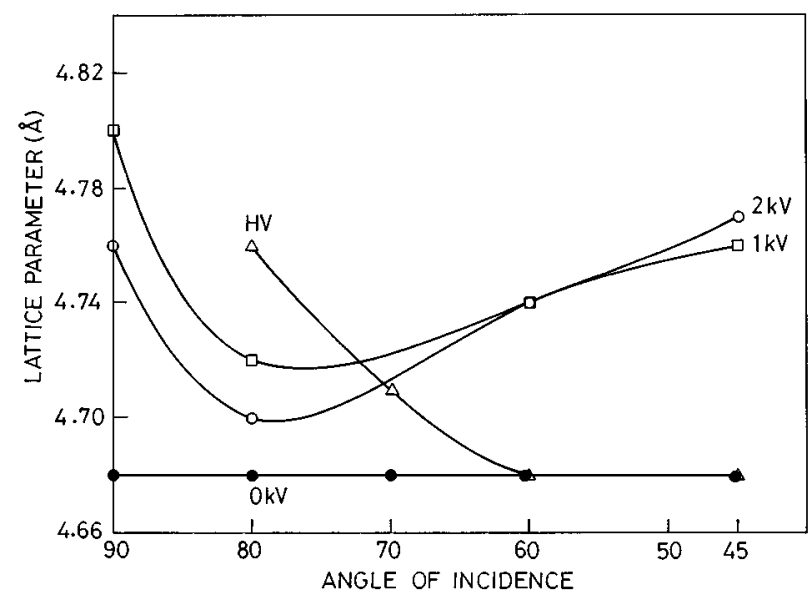

FIG. 4. Variation in the $c$ parameter of the films with the angle of ion incidence for given bias voltages.

model also seems to predict the observed experimental behavior.

It is well known that films deposited at oblique angles of vapor incidence exhibit self-shadowing, which, apart from other growth related problems, results in high tensile stress. A plot of variation of stress in the films with bias voltage (Fig. 6) at different angles of ion incidence shows that the films exhibit an increase in stress up to a critical bias voltage and decrease beyond that and that in all cases the stress in films deposited without the bias is lower than those deposited with ion bombardment. This can be attributed to the fact that the films cannot support high tensile stress and hence show a decrease beyond the critical value. Fabis, Cooke, and McDonough ${ }^{10}$ have also observed that film stress is essentially tensile at $45^{\circ}$, whereas at $0^{\circ}$ it is compressive, which approaches zero at higher values of pressure. They have observed a restricted fiber growth in the films deposited at oblique angle of incidence.

Scanning electron micrographs of the cross section of the films deposited in high vacuum at different angles of vapour incidence are shown in Fig. 7. It is evident from these micrographs that as the angle of incidence increases the films

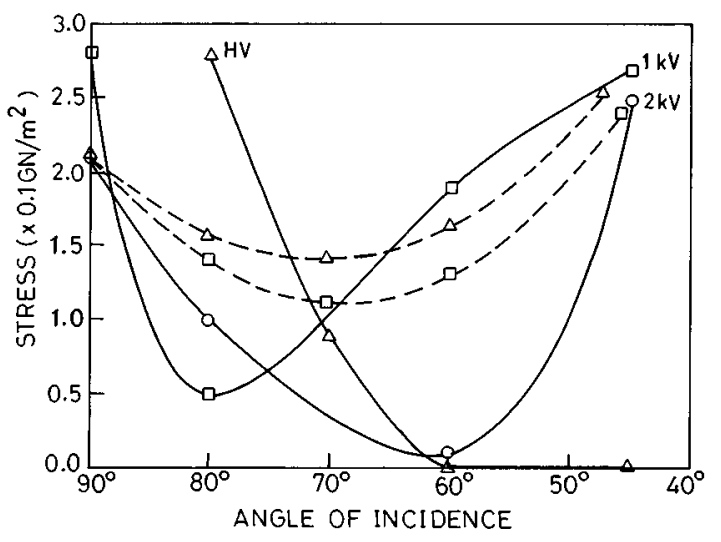

FIG. 5. Variation in stress with the angle of ion incidence for given bias voltages. 


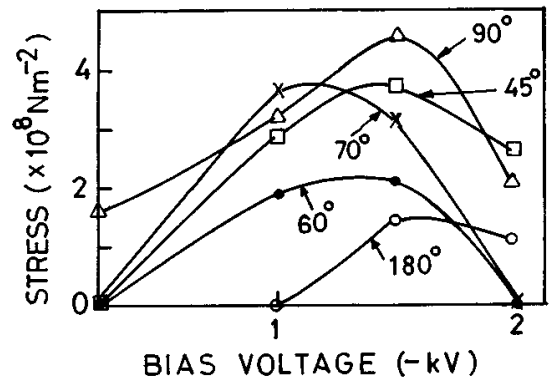

FIG. 6. Variation in stress with the bias voltage for given angles of ion incidence.

show an increasingly columnar microstructure with the column angle depending roughly on the angle of incidence of vapor. The films are mainly porous and the structure corresponds to the zone I type microstructure according to the model proposed by Messier, Giri, and Roy. ${ }^{19}$ This also correlates well with the stress behavior shown in Fig. 5. Similarly, at a bias of $2 \mathrm{kV}$ the films continue to show a textured growth with an increase in the angle of incidence (Fig. 8).

There are, however, two points of significance in these micrographs. The first point which is clear is that the films appear to have higher density than the high vacuum evaporated films. Second, the texturing of the films is very prominent up to $60^{\circ}$ incidence after which the texturing is not so evident. This is the direct consequence of the fact that sputtering yield reduces relative to the ion reflection coefficient beyond certain angles of incidence, normally in the range $60^{\circ}-80^{\circ}$, causing a decrease in texture. The films also seem to show dense fibrous growth as the bias voltage is increased and seems to correspond to the zone $T$ type of growth. ${ }^{19}$ This is further evident from Fig. 9 where the cross section of the films deposited at a constant angle of $60^{\circ}$ is shown as a function of increase in bias voltage. It can be seen that the films become denser with an increase in bias voltage and show a dense fibrous microstructure as the bias voltage is increased.

Significantly, although the films become dense, independent of deposition parameters they show some amount of porosity. This is manifested in the stress behavior, which is tensile, independent of deposition parameters.

\section{B. Discussion}

The effect of obliquely incident vapors is now a reasonably well understood phenomenon. Dirks and Leamy ${ }^{20}$ were one of the earliest workers to report on the microstructure of obliquely incident vapor deposited thin films. They observed that the inclination of columns, $\beta$, in the films could be related to the angle of vapor incidence, $\alpha$, by a simple tangent rule:

$$
\tan \alpha=2 \tan \beta .
$$

This behavior has been observed to a limited extent in the vapor deposited thin films shown in Fig. 7. Although the inclination at $30^{\circ}$ is very pronounced, at other angles it is not
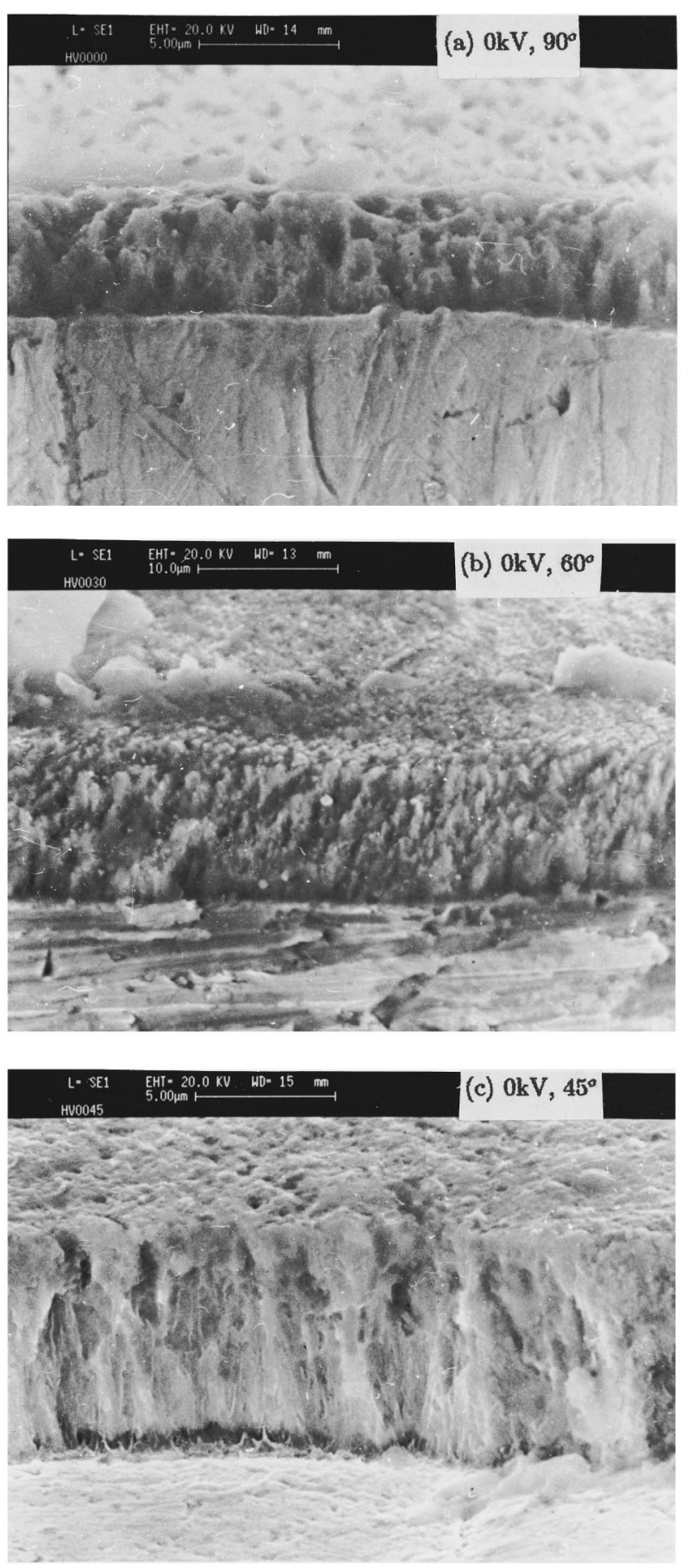

FIG. 7. Scanning electron micrograph showing the cross section of the films deposited in high vacuum without bias at given angles of vapor incidence.

so. However, the columns do seem to change their inclination with the increase in the angle of vapor incidence.

The variation in sputtering yield, briefly mentioned earlier, with increase in the angle of ion incidence, is known to reach a peak at around $60^{\circ}$ for most materials, beyond which ion reflection takes over. Oeschner ${ }^{16}$ and Rosendaal ${ }^{17}$ have reported extensively on this and a typical curve is shown in Fig. 10. It is seen that the variation in peak intensity is very 

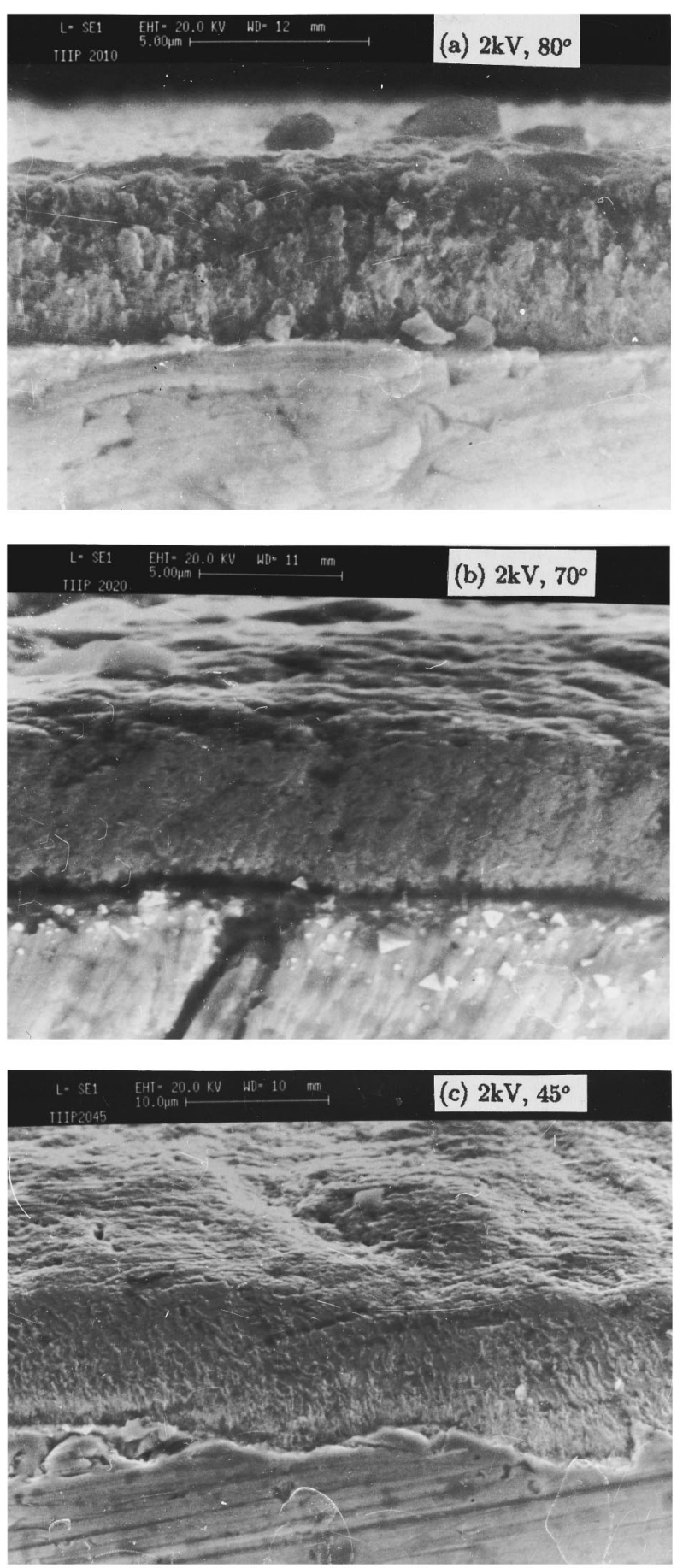

FIG. 8. Scanning electron micrograph showing the cross section of the films deposited at a bias voltage of $2 \mathrm{kV}$ at given angles of ion incidence.

similar to that of sputtering yield with the angle of incidence. A remarkable feature of the crystalline behavior is the absence of any orientation other than (002) in the films. Although only powder x-ray diffractometry has been used in the present study, it does indicate a strong preferred orientation for the films. In earlier work done by Gunasekhar et al. ${ }^{13}$ on diode ion plated Ti films deposited onto $\mathrm{Cu}$ substrates it was found that the films showed (001) reflections
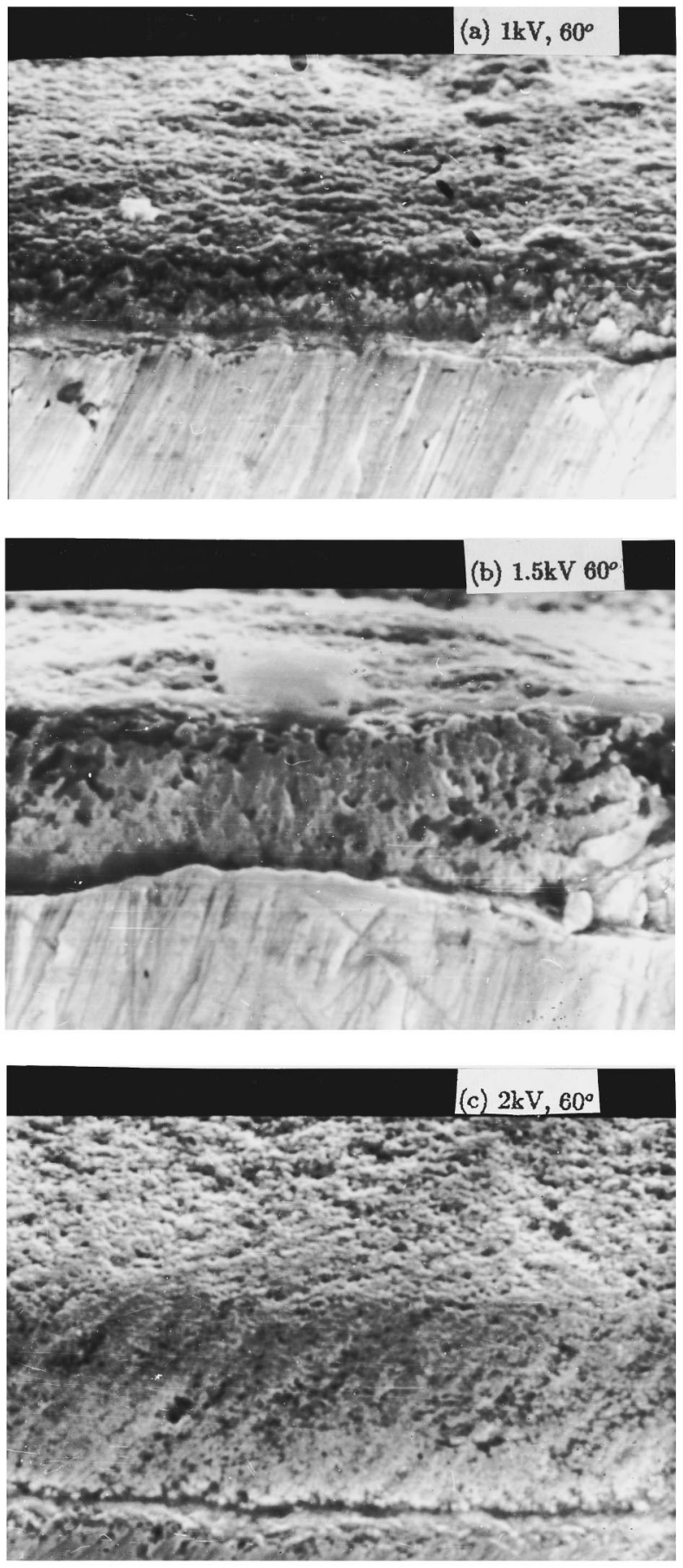

FIG. 9. Scanning electron micrograph showing the cross section of the films deposited at angle of $60^{\circ}$ and different bias voltages.

when deposited at low deposition rates and low bombardment conditions. The films then showed a transition to a preferred (011) orientation when high deposition rates, bias voltages, and current densities were used. In contrast to the earlier observations it has been found in the present investigation that upon introduction of the third electrode, the thermionic filament, the films continue to show (001) reflection even at the high bias voltage of $2 \mathrm{kV}$, a high rate of deposition of $0.3-0.6 \mu \mathrm{m} / \mathrm{min}$, and high current. The earlier work 


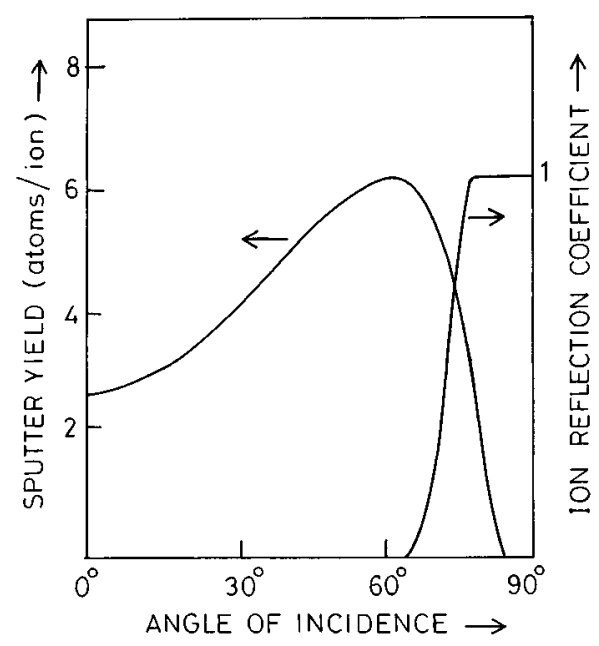

FIG. 10. Schematic representation of the variation in sputtering yield and ion reflection coefficient with the angle of ion incidence.

had reported a correlation between the orientation and compressive stress in the films and also channeling as a probable explanation for the observed orientation effects.

One of the theories postulated to explain the degree of orientational order in films deposited using non-normal ion incidence is the difference in sputtering yields of grains aligned in the direction of ion incidence and those that are misaligned. ${ }^{7,8,17}$ Rosendaal $^{17}$ has observed differences as high as a factor of 5 in these two values. The theory goes on further to invoke channeling effects to explain preferred orientation in these films. However, in $\mathrm{Ti}$ it is known that the (001) direction is the closest packed direction and thus the probability of channeling occuring in this direction is very low. Therefore, although a difference in sputtering yields could be a factor determining the (002) orientation of the films in the present study, it does not appear to be a sufficient factor in explaining the observed behavior.

To explain the observed behavior the theory of abnormal grain growth can be employed. ${ }^{21-23}$ Secondary or abnormal grain growth in thin films can be defined as the process in which some grains grow faster and, consequently, at the expense of other grains that are consumed during the process of growth. This results in large grain sizes and a smaller number of grains as opposed to normal grain growth wherein all grains grow at the same rate; hence final grains are smaller in size and larger in number. It has been found that the driving force can be related to the surface energy anisotropy, the normal grain boundary energy $\gamma_{n}$ and a barrier to growth represented by the secondary grain boundary energy, $\gamma_{\mathrm{gb}}$. These are the first, second, and third terms respectively, in Eq. (2) below: ${ }^{21-23}$

$$
\Delta F=-2(\Delta \gamma) / h-\beta \gamma_{\mathrm{gb}} / r_{n}+2 \gamma_{\mathrm{gb}} / r_{s},
$$

where $F$ is the driving force for grain growth, $h$ is the thickness of the films, and $r_{n}$ and $r_{s}$ are the normal and secondary grain sizes, respectively.

This model used on amorphous silicon films that undergo crystallization on annealing predicts that surface energy an- isotropy can play a dominant role in the initial formation of secondary grains since surface energy is a strong function of crystallographic orientations. From this model it follows that secondary grains will predominantly show orientations in a particular direction, determined by the minimum in surface energy. Therefore films will show preferred crystallographic orientations as a consequence of secondary grain growth. In other words, the films will eventually show only those orientations or crystallographic texture that have minimum surface energy anisotropy. In the present case, it is thus probable that the growth of films in a preferred (002) direction is surface energy driven because it is the closest packed direction and thus corresponds to a minimum surface energy. This mechanism appears to act in competition with the sputter yield mechanism to give the observed preferred orientation.

Although most plasma deposited films show a transition from tensile to compressive stress under different processing conditions such as pressure or bias voltage, it is interesting to see that the films in the present study show only tensile stress independent of deposition conditions. A number of models are currently in use to explain tensile stresses in thin films. ${ }^{18}$ For example, tensile stress in low melting point metals has been attributed to a phase change, amorphous to crystalline, induced either by heating the film or depositing the film above its crystallization temperature. This is obviously not so in the present case. A buried layer mechanism has also been used for explaining stress behavior with variation in substrate temperature. This again, however, is not applicable to the present case because there are no temperature variations. The most applicable model is the grain boundary relaxation model, which predicts a inverse dependence of the stress on grain size. The origin of stress in this model is believed to be the interatomic forces acting across the gaps between adjacent grains causing an elastic deformation or relaxation of the boundary walls. The deformation is further believed to be balanced by the forces imposed by the constraint exerted by the adhesion of the film to the substrate. The micrographs shown in Figs. 7-9 seem to indicate grain boundary relaxation both across the thickness (indicated by expanding columns) and the resulting spatial variation across the plane of the film. Stress is thus related to the elastic constants and a relaxation distance $\Delta$ which in most cases is equal to the ionic radius.

The final equation is of the form: ${ }^{19}$

$$
\sigma=[E /(1-\nu)](\Delta / d)
$$

where $\sigma$ is the stress, $E$ the Young's modulus, $\nu$ the Poisson ratio, $\Delta$ the ionic radius, and $d$ the calculated grain size.

It can be observed from Fig. 5 that the model also predicts a similar kind of a behavior for stress with the angle of ion incidence as that calculated from x-ray patterns. The values are slightly higher, mainly due to errors in assuming bulk elastic constants and the inherent limitation in calculation from the full width at half-maximum of the peak.

The above discussion clearly indicates that films deposited at an oblique angle of ion incidence in a triode ion plating configuration exhibit a rich array of variations in both 
structure and microstructure. On the positive side it appears that the angle of incidence is sufficient to achieve large variations in properties such as structure, stress, grain size, etc. However, gross properties such as hardness, wear, and friction have yet to be evaluated to prove the point conclusively. On the negative side, it appears that small changes in the curvature of substrates or even large surface area substrates would show variations in properties across their surfaces.

\section{SUMMARY}

In summary, Ti films deposited at different angles of ion incidence using triode ion plating have been studied for their structure, grain size, stress, and lattice parameter. It has been found that the obliquely incident deposited films show anisotropy in their properties which could be exploited.

${ }^{1}$ J. A. Thornton, Thin Solid Films 40, 335 (1977).

${ }^{2}$ D. W. Hoffmann and J. A. Thornton, Thin Solid Films 64, 111 (1979).

${ }^{3}$ R. N. Castellano, M. R. Notis, and G. W. Simmons, Vacuum 27, 109 (1977).

${ }^{4}$ D. Dobrev, Thin Solid Films 92, 41 (1980).

${ }^{5}$ R. A. Roy and D. S. Yee, in Handbook of Ion Beam Processing Technology, edited by J. J. Cuomo, S. Rossnagel, and H. R. Kaufman (Noyes, Park Ridge, NJ, 1989), p. 94.
${ }^{6}$ R. M. Bradley, in Ref. 5, p. 300.

${ }^{7}$ L. S. Yu, J. M. E. Harper, J. J. Cuomo, and D. A. Smith, Appl. Phys. Lett. 47, 932 (1985).

${ }^{8}$ L. S. Yu, J. M. E. Harper, J. J. Cuomo, and D. A. Smith, J. Vac. Sci. Technol. A 4, 443 (1986).

${ }^{9}$ J. J. Cuomo, D. L. Pappas, R. Lossy, J. P. Doyle, J. Bruley, G. W. DiBello, and W. Krakow, J. Vac. Sci. Technol. A 10, 3414 (1992).

${ }^{10}$ P. M. Fabis, R. A. Cooke, and S. McDonough, J. Vac. Sci. Technol. A 8, 3809 (1990).

${ }^{11}$ W. Ensinger, Surf. Coat. Technol. 65, 90 (1994).

${ }^{12}$ Y. Andoh, K. Ogata, Y. Yamaka, and S. Sakai, Nucl. Instrum. Methods B 39, 70 (1989).

${ }^{13}$ K. R. Gunasekhar, S. Srinivasulu, M. Swarnalatha, M. Ghanashyam Krishna, and S. Mohan, Thin Solid Films 252, 7 (1994).

${ }^{14}$ K. R. Gunasekhar and S. Mohan, Vacuum 42, 661 (1991).

${ }^{15}$ M. Ghanashyam Krishna, S. Kanakaraju, K. Narasimha Rao, and S. Mohan, Mater. Sci. Eng. B 21, 10 (1993).

${ }^{16}$ H. Oeschner, Appl. Phys. 8, 185 (1975).

${ }^{17}$ H. E. Rosendaal, in Sputtering by Particle Bombardment, edited by R. Behrisch (Springer, Berlin, 1980), Vol. 47, Chap. 5.

${ }^{18}$ H. Windischmann, Crit. Rev. Solid State Mater. Sci. 17, 547 (1992).

${ }^{19}$ R. Messier, A. P. Giri, and R. A. Roy, J. Vac. Sci. Technol. A 2, 500 (1984).

${ }^{20}$ A. G. Dirks and H. J. Leamy, Thin Solid Films 47, 219 (1977).

${ }^{21}$ C. V. Thompson, J. Appl. Phys. 58, 763 (1985).

${ }^{22}$ C. V. Thompson, J. A. Floro, and H. I. Smith, J. Appl. Phys. 67, 4099 (1990).

${ }^{23}$ C. V. Thompson, Annu. Rev. Mater. Sci. 20, 245 (1990). 